\title{
An Improved Case of Autism as Revealed by PET CT Scan in Patient Transplanted with Autologous Bone Marrow Derived Mononuclear Cells
}

Alok Sharma1, Prerna Badhe1, Nandini Gokulchandran¹, Pooja Kulkarni², Priti Mishra ${ }^{3}$, Akshata Shetty ${ }^{3}$ and Hemangi Sane ${ }^{2}$

${ }^{1}$ Department of Medical Services and Clinical Research, NeuroGen Brain and Spine Institute, India

${ }^{2}$ Department of Research \& Development, NeuroGen Brain and Spine Institute, India

${ }^{3}$ Department of NeuroRehabilitation, NeuroGen Brain and Spine Institute, India

\begin{abstract}
Autism, the most severe form of autism spectrum disorder (ASD), is a complex neurodevelopmental disorder, characterized by language developmental delay, social skills impairment, communication problems, and restricted, repetitive, and stereotyped patterns of behavior. There is no cure for Autism; hence therapies and behavioral interventions are designed to remedy specific symptoms. We used autologous bone marrow derived mononuclear cells intrathecally in a $14 \mathrm{yr}$ old boy with severe autism to improve the quality of life. At six months, follow up after therapy the general impression on clinical assessment showed mild autism. It is exciting to see symptomatic improvement with shift on Childhood Autism Rating Scale (CARS) from 42.5 (Severely Autistic) to 23.5 (Non Autistic), which was also visualized as enhanced PET scan brain function. All these improvements have led to improved quality of life of the patient as well as the family. Several incurable neurological disorders have shown benefits with cellular therapy thus, autism should be explored as an indication and nuclear imaging can be used to study its effects.
\end{abstract}

Keywords: Autism; Autologous; Bone marrow; Mononuclear cells; PET

\section{Introduction}

Autism is a neurodevelopmental, disorder with a multidimensional presentation. It isnoticeable by parents at the age of 3 years due to delayed or abnormal language development, deficits in social interaction, lack of eye contact, hyperactivity and repetitive behaviors and interests [1]. Autism is the most severe form of autism spectrum disorder (ASD), while other conditions along the spectrum include mild forms known as Asperger's syndrome, childhood disintegrative disorder and pervasive developmental disorder. 1 in every 166 children is estimated to have autism [2]. Although outcomes are specific, behavioral characteristics change over time. Most autistic children remain within the spectrum as adults and continue to experience difficulty with independent living, employment, social relationships, and mental health. Since autism is incurable, chronic long term management is required and can become progressively difficult to handle for parents. The primary goal of the treatment is to improve the quality of life of the patient by minimizing the core features and associated deficits and maximizing functional independence. Facilitating development and learning, promoting socialization, reducing maladaptive behaviors, and educating and supporting families can help accomplish these goals [3-5].

\section{Material and Methods}

\section{Case presentation}

Herein, we present a 14 year old boy with autism, who had birth history of Full Term - C - section delivery followed by normal motor milestones but delayed speech with lack of social interactions and emotional development. He was hyperactive with behavioral issues like engaging in self-injurious behavior, self-scratching and hyper aggression. He had normal vision and hearing with slurred speech. $\mathrm{He}$ was disoriented in time, but identified places and people. There was no history of seizures. Neurologically, he had normal muscle tone and power in trunk and limb muscles. Functionally, he was independent for most of the daily activities but needed assistance in fine motor activities. In spite of regular rehabilitation since the age of 6 years, he showed no improvements with respect to behavior and social interaction.
His intelligent quotient (IQ) was found to be 64 and Childhood Autism Rating Scale (CARS) score was 42.5 which is categorized as severely autistic.

The molecular diagnostic test of fragile $\mathrm{X}$ done using southern blot was negative. MRI Brain showed no significant intracranial abnormality and EEG showed bilateral episodic sharp and slow wave abnormalities. The brain PET scan showed moderately reduced metabolic activity in right broca's, right insula, right lateral temporal pole, right calcarine, both basal ganglia and left medial prefrontal. It also showed mildly reduced metabolic activity in right parietal, right sensory motor and right Wernicke's.

He underwent autologous bone marrow derived mononuclear cell transplantation. Our protocol is based on the inclusion criterion as per the World Medical Associations Helsinki declaration [6]. It has been reviewed and approved by the Institutional committee for Stem cell Research and Therapy (IC-SCRT). The patient's parents were informed about the procedure and a duly filled informed consent form was obtained from them. $300 \mathrm{mcg}$ of G-CSF injections were administrated 48 hours and 24 hours before bone marrow derived mononuclear cell transplantation, to stimulate CD34+ cells and increase their survival and multiplication. Bone marrow $(100 \mathrm{ml})$ was aspirated from the iliac bone. Mononuclear cells (MNCs) were obtained using density gradient separation method. Viable count of the isolated MNCs was taken and was found to be about $98 \%$. The MNCs were checked for CD34+ by

*Corresponding author: Pooja Kulkarni, NeuroGen Brain and Spine Institute SuranaSethia Hospital and Research centre, Suman Nagar, Sion-Trombay Road, Chembur, Mumbai-400071, India, Tel: 022-25281610/25283706; E-mail: poojakul28@gmail.com

Received April 23, 2013; Accepted May 18, 2013; Published May 20, 2013

Citation: Sharma A, Badhe P, Gokulchandran N, Kulkarni P, Mishra P, et al. (2013) An Improved Case of Autism as Revealed by PET CT Scan in Patient Transplanted with Autologous Bone Marrow Derived Mononuclear Cells. J Stem Cell Res Ther 3 : 139. doi:10.4172/2157-7633.1000139

Copyright: @ 2013 Sharma A, et al. This is an open-access article distributed unde the terms of the Creative Commons Attribution License, which permits unrestricted use, distribution, and reproduction in any medium, provided the original author and source are credited. 
Citation: Sharma A, Badhe P, Gokulchandran N, Kulkarni P, Mishra P, et al. (2013) An Improved Case of Autism as Revealed by PET CT Scan in Patient Transplanted with Autologous Bone Marrow Derived Mononuclear Cells. J Stem Cell Res Ther 3: 139. doi:10.4172/21577633.1000139

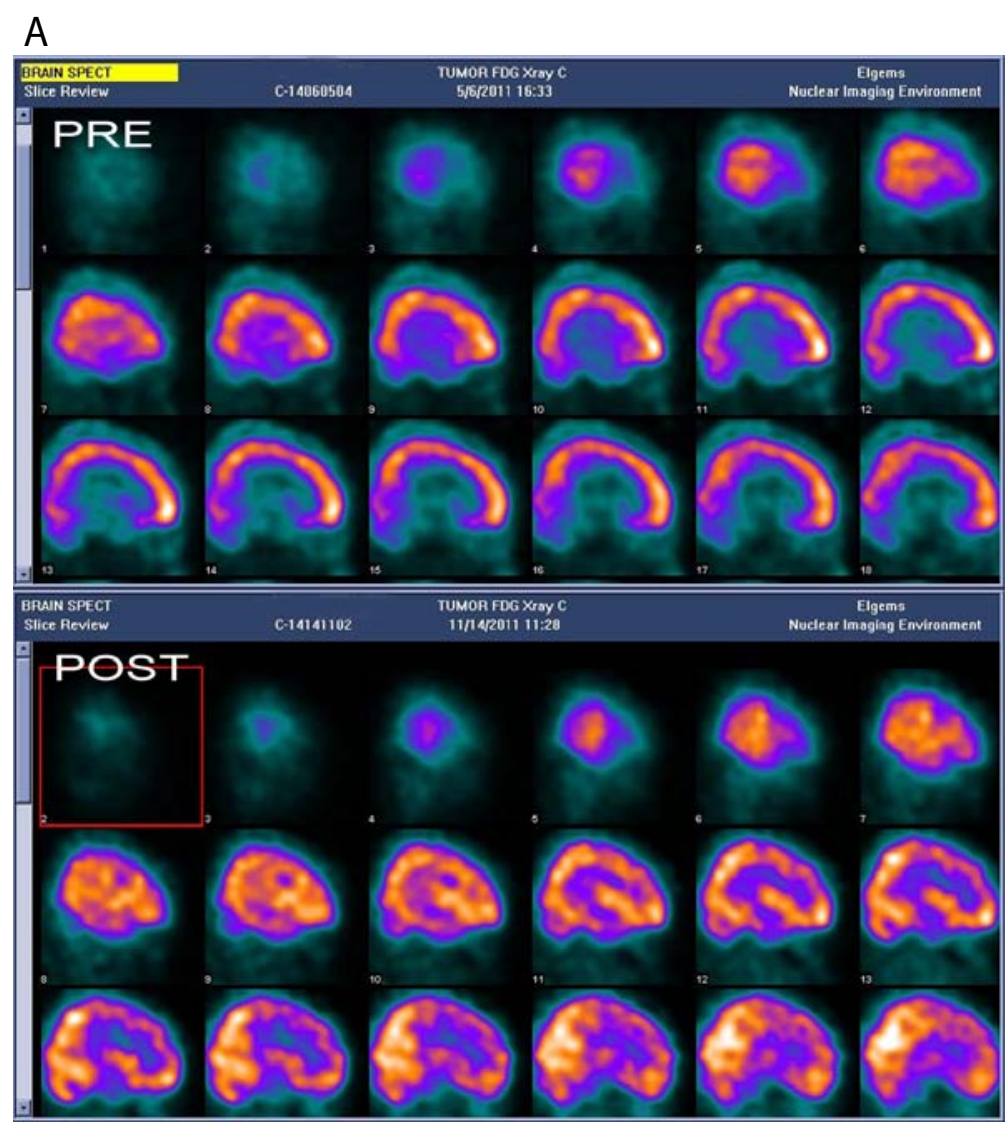

B

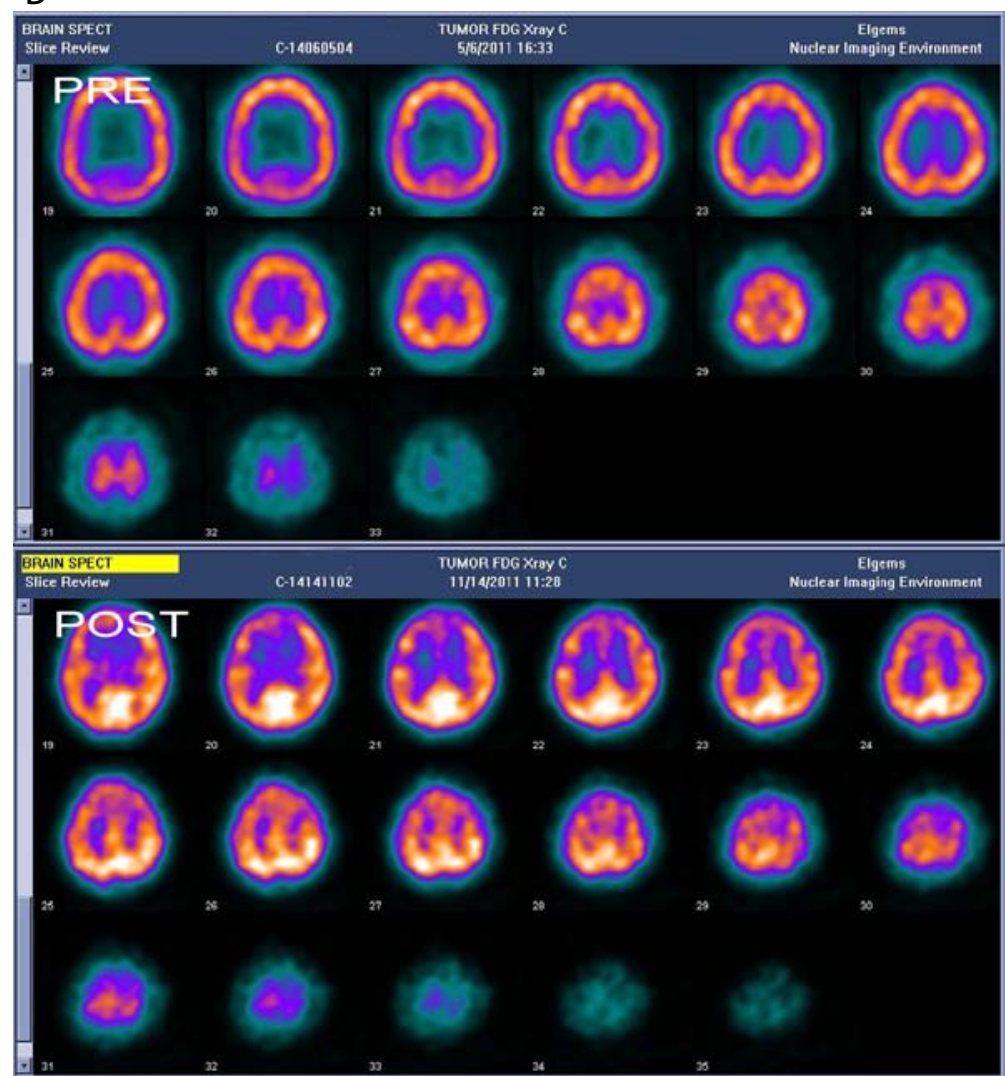


Citation: Sharma A, Badhe P, Gokulchandran N, Kulkarni P, Mishra P, et al. (2013) An Improved Case of Autism as Revealed by PET CT Scan in Patient Transplanted with Autologous Bone Marrow Derived Mononuclear Cells. J Stem Cell Res Ther 3: 139. doi:10.4172/21577633.1000139

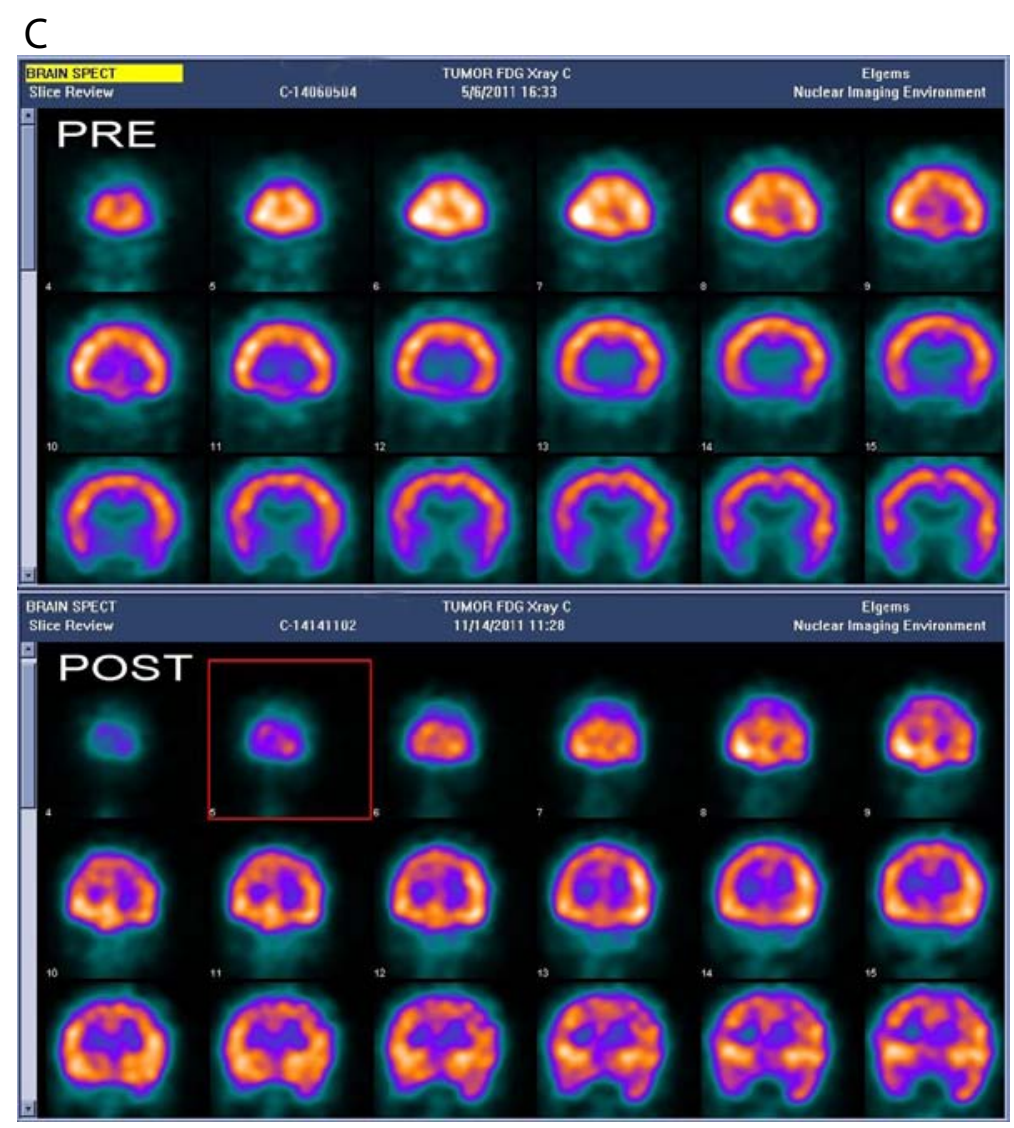

Figure 1: A, B, C: The Pre and the Post cell therapy PET CT scans. Comparative study of pre and post cell therapy PET CT scan shows increased FDG uptake in bilateral temporal lobes and bilateral calcarine cortices with mild increased uptake in left medial pre-frontal cortex as visualized below in the post scans.

FACS analysis and was determined to be $1.92 \%$. Approximately 56 $\times 106$ MNCs were immediately injected post separation, intrathecally in L4-L5 using a lumbar puncture needle and catheter. The patient was also given rehabilitation therapy which included occupational therapy and psychological therapy. Rehabilitation interventions seek to promote recovery \& independence through neurofacilitation. During rehabilitation sessions, effective motor learning strategies with task oriented training, for real life environment were utilized \& successful attainment of functional outcomes were achieved. He was evaluated at regular intervals of three and six months. He was reassessed by repeating CARS and PET scan of brain after 6 months.

\section{Results}

After the procedure, the patient had no side effects. The patient showed some immediate improvements within a week and significant improvements over a period of six months to one year.

Within a week, there was improvement in his eye contact and attention. His hand-writing and fine motor activities like buttoning had improved significantly. On follow up after six months, further improvements were observed in his behavior with respect to social interaction and emotions. Aggression in activities and hyperactivity had reduced by 45 to $50 \%$. Improvements in impulse control, reading skills, tracing, recognition of all shapes and following commands were noted. His score on CARS reduced from 42.5 (Severely autistic) to 23.5 (Non-Autistic) but the general impression on clinical assessment showed mild autism.
On repeating brain PET scan after 6 months, there were improvements recorded in comparison with previous report. Comparative study of previous and post stem cell therapy PET CT scan showed markedly increased uptake in bilateral temporal lobes and bilateral calcarine cortices with mild increased uptake in left medial pre-frontal cortex. All the views; saggital, transverse and coronal views (Figure 1) showed the improvements.

At one year follow up, he was found to interact more with his peers. Peer activity had increased significantly. New task learning abilities had improved which was noticed due to increased participation in household work. Comprehension and ability to follow commands had improved significantly. He had developed self insight and appropriate emotional response.

\section{Discussion}

The exact etiology of autism is not known, but it is likely to result from a complex combination of genetic, environmental, and immunological factors [7]. Current available treatments for autism can be divided into behavioral, nutritional, and pharmacological options, in addition to individual and family psychotherapy and other nonpharmacologic interventions [8]. However, there is no defined standard approach [9]. Currently, numerous clinical trials are being conducted with interventions ranging from hyperbaric oxygen, to administration of zinc, to drugs exhibiting anti-inflammatory properties.

Stem cell therapy offers great promise for the treatment of autism. Although several neurophysiological alterations have been correlated 
Citation: Sharma A, Badhe P, Gokulchandran N, Kulkarni P, Mishra P, et al. (2013) An Improved Case of Autism as Revealed by PET CT Scan in Patient Transplanted with Autologous Bone Marrow Derived Mononuclear Cells. J Stem Cell Res Ther 3: 139. doi:10.4172/21577633.1000139

Page 4 of 4

with autism, immune dysfunction and neural hypoperfusion appear to be broadly consistent. The association of altered inflammatory responses and hypoperfusion with symptology is reported, which suggests its causative role [10]. The BMMNCs are comprised of a variety of cells which includes mesenchymal stem cells (MSCs), hematopoietic stem cells, tissue specific progenitor cells and stromal cells. Mesenchymal stem cell have an ability to modulate the immune system and restore the altered brain organization with unique property of homing, wherein the cells migrate to the site of injury and carry out the repair process [11]. The various stem cells in MNC enhance angiogenesis by producing signaling molecules such as vascular endothelial growth factor (VEGF) and fibroblast growth factor (FGF2). They also promote tissue remodeling, prevent apoptosis, decrease inflammation and activate the satellite cells [12]. We have previously published data wherein autologous mononuclear cell transplantation was carried out in children with various incurable neurological disorders including autism, cerebral palsy and mental retardation. Improvements were reported in quality of life of these patients [12-14]. On repeating and comparing the PET scan of brain after six months post therapy, significant changes were recorded and correlated to symptomatic improvements. In numerous studies the areas affected by hypoperfusion seem to correlate with regions of the brain that are responsible for dysfunctions in autism. For example, specific temporal lobe areas associated with face recognition, social interaction, and language comprehension, have been demonstrated to be hypoperfused in autism [10]. The functions of insulae include emotional processing, empathy, perception, motor control, selfawareness, cognitive functioning, and interpersonal experience. In our case these areas showed improvements on PET scan of brain. So the clinical and radiological improvements are well correlated.

Hence, we hypothesize that the cumulative effect of various cells in MNCs showed significant clinical improvements in this case and has altered the course of disease. We conclude, though single case, that intrathecal transplantation of autologous bone marrow derived mononuclear cells is safe, feasible; and may be beneficial in autism. The large clinical studies are an immediate need to fully explore its potential in autism and nuclear imaging can be used to study its effects.

\section{References}

1. Hu VW, Sarachana T, Kim KS, Nguyen A, Kulkarni S, et al. (2009) Gene expression profiling differentiates autism case-controls and phenotypic variants of autism spectrum disorders: evidence for circadian rhythm dysfunction in severe autism. Autism Res 2: 78-97.

2. Fombonne E (2005) Epidemiology of autistic disorder and other pervasive developmental disorders. J Clin Psychiatry 66: 3-8.

3. Howlin P (2005) Outcomes in autism spectrum disorders: Handbook of Autism and Pervasive Developmental Disorders (3rd ed), Hoboken, NJ: John Wiley $\&$ Sons.

4. Howlin P, Goode S, Hutton J, Rutter M (2004) Adult outcome for children with autism. J Child Psychol Psychiatry 45: 212-229.

5. Seltzer MM, Shattuck P, Abbeduto L, Greenberg JS (2004) Trajectory of development in adolescents and adults with autism. Ment Retard Dev Disabi Res Rev 10: 234- 247.

6. Carlson RV, Boyd KM, Webb DJ (2004) The Revision of The Declaration of Helsinki: Past, Present And Future. Br J Clin Pharmacol 57: 695-713.

7. Persico AM and Bourgeron T (2006) Searching for ways out of the autismmaze: genetic, epigenetic and environmental clues. Trends in Neurosciences 29 : 349-358.

8. Reiersen AM, Todd RD (2008) Co-occurrence of ADHD and autism spectrum disorders: phenomenology and treatment. Expert Rev Neurother 8: 657-669.

9. Ospina MB, Seida JK, Clark B, Karkhaneh M, Hartling L, et al. (2008) Behavioural and developmental interventions for autism spectrum disorder: a clinical systematic review. PLoS One 3.

10. Ichim TE, Solano F, Glenn E, Morales F, Smith L, et al. (2007) Stem cel therapy for autism. J Transl Med 27: 30.

11. Li L and Jiang J (2011) Regulatory factors of mesenchymal stem cell migration into injured tissues and their signal transduction mechanisms. Front Med 5 : 33-39.

12. Sharma A, Gokulchandran N, Chopra G, Kulkarni P, Lohia M, et al. (2012) Administration of autologous bone marrow-derived mononuclear cells in children with incurable neurological disorders and injury is safe and improves their quality of life. Cell Transplantat 21.

13. Sharma A, Kulkarni P, Sane H, Gokulchandran N, Badhe P, et al. (2012) Positron Emission Tomography- Computed Tomography scan captures the effects of cellular therapy in a case of cerebral palsy. Journal of clinical case reports. J Clin Case Rep 2: 195

14. Sharma A, Sane H, Paranjape A, Gokulchandran N, Kulkarni P, et al (2013) Positron Emission Tomography - Computer Tomography scan used as a monitoring tool following cellular therapy in Cerebral Palsy and Mental Retardation - A Case Report. Case Rep Neurol Med. 